\title{
The Glucocorticoid-Inducible GVG System Causes Severe Growth Defects in Both Root and Shoot of the Model Legume Lotus japonicus
}

\author{
Stig Uggerhøj Andersen, Cristina Cvitanich, Birgit Kristine Hougaard, Andreas Roussis, \\ Mette Grønlund, Dorthe Bødker Jensen, Line Askou Frøkjær, and Erik Østergaard Jensen \\ Laboratory of Gene Expression, Department of Molecular Biology, University of Aarhus, Gustav Wieds Vej 10, DK-8000 \\ Aarhus C., Denmark
}

Submitted 23 May 2003. Accepted 27 August 2003.

\begin{abstract}
During the past decade, the legume Lotus japonicus has emerged as an important model system for study of symbiotic nitrogen fixation. Controlled expression of genes involved in symbiosis from an inducible promoter at specific time points would be a valuable tool for investigating gene function in $L$. japonicus. We have attempted to study the function of the putative transcription factors $L j \mathrm{NDX}$ and LjCPP1 by expression from the GVG inducible system. This study showed that the GVG system itself causes growth disturbances in $L$. japonicus. Shoot internode elongation and root pericycle cell division are affected when the chimeric GVG transcription factor is activated. We suggest that deficient auxin signaling could cause the phenotype observed and conclude that the GVG inducible system is not well suited for use in the model legume $L$. japonicus.
\end{abstract}

Additional keywords: DEX, gibberellin, lateral root initiation, starch.

Reverse genetics is an important tool for studying gene function. Normally, two complementary approaches are used. First, the gene of interest is overexpressed from a strong constitutive promoter. Second, an attempt is made to knock down gene expression by expressing either intron-hairpin RNA or antisense RNA, likewise from a strong constitutive promoter. These approaches work well for many genes. In some cases, however, the altered gene expression levels caused by the transgenes are lethal, making it impossible to recover transgenic plants.

To address this problem, and to allow study of gene function at different developmental stages, several inducible systems have been made, which allow control of transgene expression by exogenous application of various substances to the plant (Zuo and Chua 2000).

One such system, GVG (Aoyama and Chua, 1997), uses glucocorticoid hormones as the inducing agent. Being tightly controlled and highly inducible, the GVG system seemed to fulfill the requirements for an attractive system. It is a two-component system where the chimeric GVG transcription factor is constitutively expressed from a cauliflower mosaic virus $35 \mathrm{~S}$ (CaMV35S) promoter. The GVG transcription factor consists of the GAL4 DNA-binding domain from yeast, the VP16 transactivating domain from Herpes simplex virus, and the regulatory domain from rat glucocorticoid receptor (GR), which responds to the synthetic glucocorticoid dexamethasone

Corresponding author: Erik Østergaard Jensen; E-mail: eoj@mb.au.dk
(DEX). Transgene expression is controlled by a promoter which contains a binding site for the GAL4 DNA binding domain (Aoyama and Chua 1997). No adverse effects of the GVG system had been reported at the time these experiments were initiated. Later, however, growth defects in empty vector lines were reported in both Arabidopsis spp. and rice (Kang et al. 1999; Ouwerkerk et al. 2001; Sanchez and Chua 2001; Yamamoto et al. 2001).

The GVG system was used in the model legume L. japonicus (Handberg and Stougaard 1992) to investigate the function of the putative transcription factors CPP1 and NDX, for which constitutive expression of sense and antisense RNA, respectively, had proved lethal. Here, we present a detailed description of the phenotype caused by activation of the chimeric GVG transcription factor in L. japonicus and suggest that altered transport of, or sensitivity to, the plant hormone auxin could cause the phenotype observed.

\section{RESULTS}

Activation of the chimeric GVG transcription factor causes shortened internode length or complete arrest of shoot growth.

We have made several transgenic plant lines where expression of the transgene is controlled by the GVG system. These lines include GVG-gus (Ggus), GVG-Ljndxl-sense (Gndxls), GVG-Ljndxl-antisense (Gndxla), GVG-Ljndx2-antisense $(G n d x 2 a)$, GVG-Ljcppl-sense (Gcppls) and GVG-Ljcpp1antisense (Gcppla) lines. Previously, we have described the cppl and $n d x$ genes (Cvitanich et al. 2000; Gronlund et al. 2003; Jorgensen et al. 1999). In total, 36 independent lines were investigated and 23 of these, 3 Ggus, 1 Gndxls, 1 Gndxla, 3 Gndx2a, 9 Gcppls, and 6 Gcppla lines, showed a stunted shoot phenotype upon DEX induction. The shoot phenotypes ranged from mildly stunted to complete arrest of shoot growth (Fig. 1). Plants with shortened internodes will be referred to as "bushy" while plants that produce cotyledons but no fully developed true leaves will be called "small."

Shoot phenotype correlates

with GVG induced transgene expression level.

The shoot phenotype varied not only between lines, but individual lines also segregated into plants displaying normal, bushy, or small phenotypes. We investigated the Ggus lines for correlation between gus expression and phenotype. The GVG system appears to be tightly regulated, because none of the uninduced controls showed staining (Fig. 2A and B), whereas 
their induced counterparts showed strong staining (Fig. 2C and D). T1 plants showed a spotty, variable staining, (Fig. 2 A through D), whereas T2 plants stained uniformly, with the strongest staining in the vascular system. Nodule primordia, but not mature nodules, stained (Fig. 2I through K). The Ggus T2 plants segregated into weakly staining, normal-looking plants (Fig. 2E and F) and strongly staining plants exhibiting growth arrest (Fig. $2 \mathrm{G}$ and $\mathrm{H}$ ). Thus, the level of transgene expression correlated with shoot phenotype.

The correlation between GUS stain and phenotype prompted us to investigate further the correlation between GVG expression, transgene expression and phenotype. For this purpose we designed different primer sets for use in quantitative reverse-transcriptase-polymerase chain reaction (QPCR) experiments. Ljcppl endogenous RNA was quantified with a primer set where the forward primer was located in the $5^{\prime}$ untranslated region of the Ljcppl gene, outside the area of the gene used for cppl sense and antisense constructs. The total amount of Ljcppl mRNA from both endogenous and transgene sources was measured with primers amplifying bases 594 to 954 from the start methionine codon of the Ljcppl mRNA (sequence to be released on publication). Transgene expression from the Gcppla construct was measured using primers where the forward primer was situated at the transcriptional start site of the minimal CaMV35S promoter located $3^{\prime}$ of the GVG response element. GVG expression level was quantified using primers amplifying GAL4 and part of the VP16 sequence of the GVG transcription factor. For normalization of RNA content, primers amplifying the ubiquitin and ATPsynthase household gene mRNAs were designed. Ubiquitin
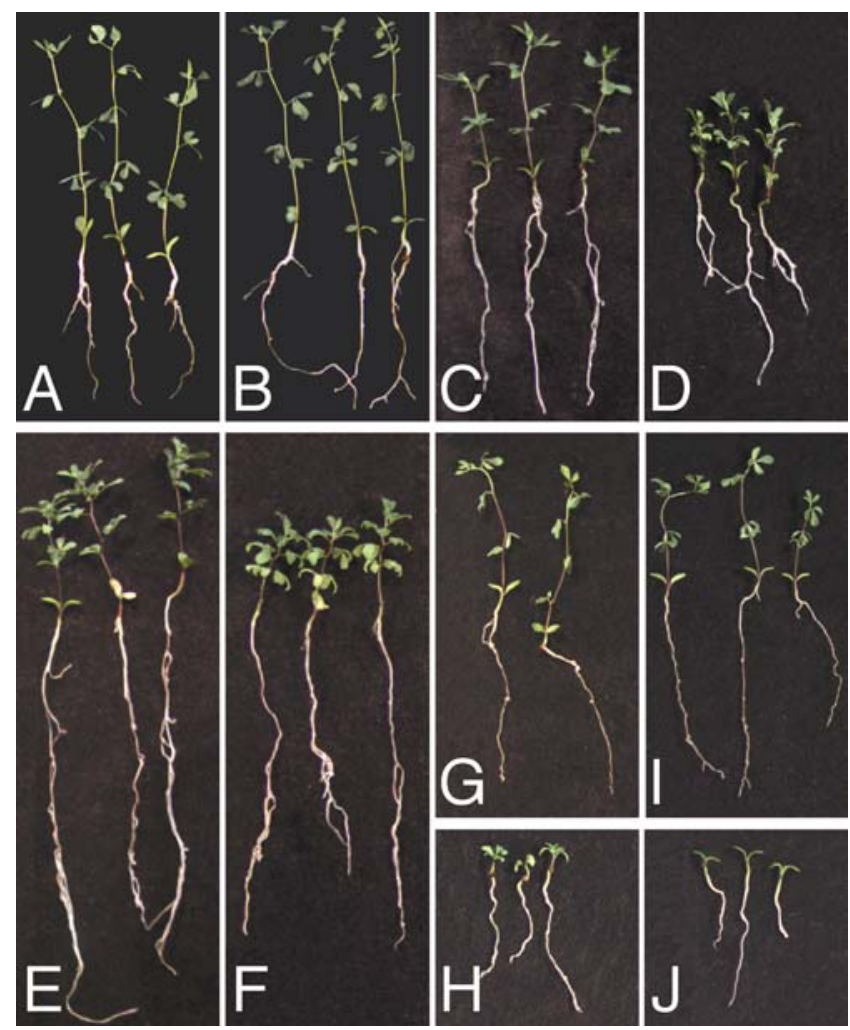

Fig. 1. Dexamethasone (DEX) induction of plants harboring GVG constructs. A and B, Wild-type plants (34 days old) inoculated with rhizobium at day 4 . The plants shown in A and B were indistinguishable. $\mathbf{C}$ and D, Ggus plants (27 days old) supplied with $10 \mathrm{mM} \mathrm{KNO}_{3}$ at day 4. E and F, Gcppla-2 plants (53 days old) supplied with $10 \mathrm{mM} \mathrm{KNO}_{3}$ at day 4 . $\mathbf{G}$ and $\mathbf{H}$, Gndx $1 a$ plants (38 days old) inoculated with rhizobium at day 21 . I and J, Gcppla-1 plants (34 days old) inoculated with rhizobium at day 21 . A, C, E, G, and I, Mock-induced plants. B, Plants induced with DEX at day 12. D, F, H, and J, Plants induced with DEX at day 4. primers amplify an mRNA encoding a putative L. japonicus ubiquitin protein precursor (accession number BF177770), whereas ATP-synthase primers amplify the L. japonicus ATPsynthase mRNA (accession number AW719841).

Transgene expression level and GVG expression level was measured for two independent Gcppls lines. DEX induction increased Ljcppl expression in the transgenic lines 5- to 20-fold (Fig. 3). While similar ubiquitin expression levels were found in wild-type (wt) plants and Gcppls plants, the Ljcppl expression level in wt plants with and without DEX treatment fell below the detection limit for quantification (data not shown). Thus, the uninduced Gcppls lines had a somewhat higher Ljcppl expression than wt plants, indicating that the GVG system is slightly leaky. The leakiness was not pronounced enough to produce a visible GUS stain in the Ggus lines (Fig. 2A through D), but became apparent when the system was probed with the highly sensitive QPCR technique. Plants with the small phenotype had a high level of Ljcppl induction. Bushy plants also were induced significantly but to a lower level, in both root and shoot, than plants displaying the small phenotype. GVG expression levels were similar in all plants tested and were not changed by DEX induction. The small plants had a slightly higher GVG expression level than bushy plants. These results confirmed that plants with the small phenotype had a high level of transgene expression, as also was seen in the Ggus lines. The results also hinted that the GVG expression level might be used as an indicator for phenotype severity. This was investigated further by measuring average GVG levels for four different transgenic lines displaying different shoot phenotypes. A comparison of GVG expression levels in four lines that were chosen for further investigation is shown in Figure 4. The Gcppla-1, Ggus, and Gndxla lines are represented by plants from the $\mathrm{T} 2$ generation, whereas the

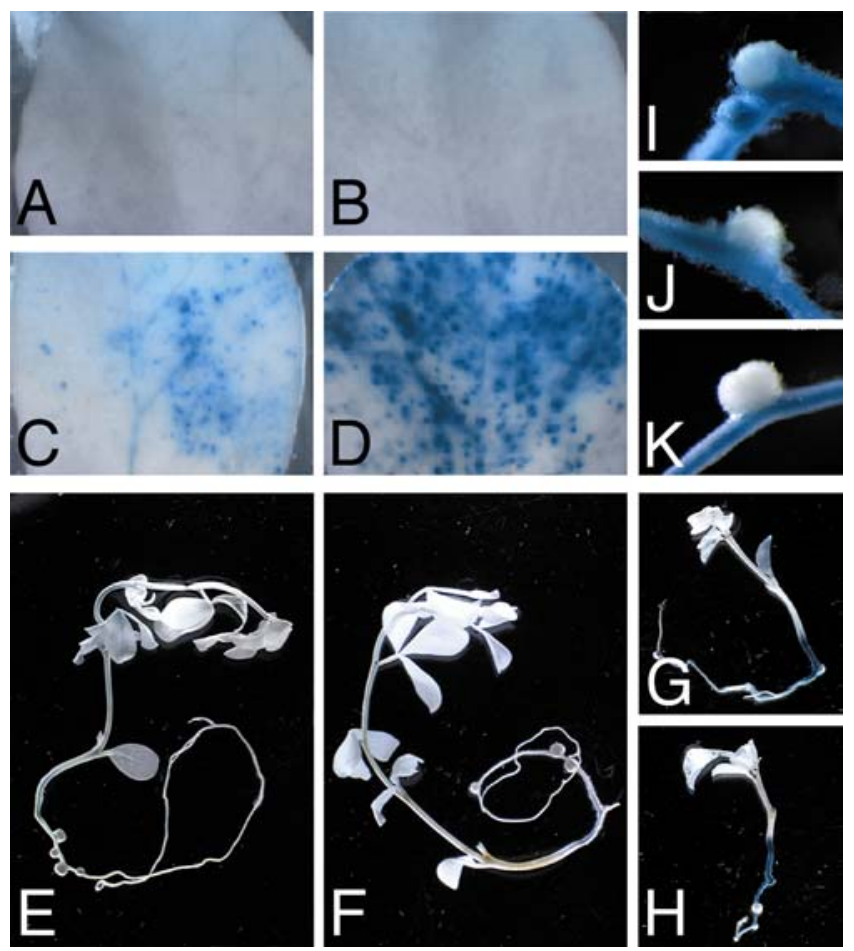

Fig. 2. Dexamethasone (DEX) induction of Ggus plants. A through D, Primary transformants. A and B, Control leaves incubated in water for $24 \mathrm{~h}$ prior to GUS staining. No control leaves showed staining. C and D, Leaves from the same plant induced with DEX for $24 \mathrm{~h}$ prior to GUS staining. E through $\mathbf{H}$, T2 generation plants (5 weeks old) grown in the presence of 30 $\mu \mathrm{M}$ DEX for 4 weeks. E and $\mathbf{F}$, Normal-looking plants exhibiting weak staining. $\mathbf{G}$ and $\mathbf{H}$, Strong staining, severely stunted plants. I through $\mathbf{K}$, Staining of nodules at different developmental stages. 
Gcppla-2 line is represented by T3 generation plants. The Gcppla-2 line was selected for study because T2 plants showed a disordered root phenotype while shoot growth was not severely affected. The T3 generation retained these characteristics and the GVG expression level in the Gcppla-2 line was significantly lower than in the T2 lines shown ( $t$ test, 95\% confidence level). The T2 lines were selected for further study in order to have lines with different shoot phenotypes and constructs for comparison. The level of GVG expression was highest in the Gcppla-1 line, which also had the most severe shoot phenotype (Fig. 1I and J), whereas the Ggus line (Fig. 1C and D) and the Gndxla line (Fig. $1 \mathrm{G}$ and $\mathrm{H}$ ) had similar GVG expression levels but very different shoot phenotypes. Even though there is a tendency that lines with the highest average GVG expression levels produce the most severe shoot phenotypes, relatively small differences in GVG expression levels can result in dramatic differences in GVGrelated phenotypes.

\section{Arrested shoot apical meristems form leaf primordia but no internode.}

Despite their stunted appearance, bushy plants produce as many sets of true leaves as their uninduced counterparts (data not shown). To investigate whether small plants produce leaf primordia, sections of the shoot meristem from a 33-day-old small plant were compared with sections of a meristem from a 5-day-old wt plant similar in size to the small plant. The wt meristem is shown in Figure 5. Here, the cotyledons frame the shoot apical meristem (SAM). Three developing true leaves are seen above the meristem, while two leaf primordia are visible on either side of the meristem. Between the SAM and the base of the cotyledons, the first internode of the plant is developing. A small side shoot can be seen at the base of the internode. The region surrounding the SAM of the small plant (Fig. 5B) also shows three developing real leaves above the SAM and a number of leaf primordia immediately around it. However, the SAM itself still is situated just above the base of the cotyledons and no internode has been formed.

The bushy phenotype caused by the GVG inducible system can be rescued by the addition of exogenous gibberellin.

We investigated whether the lack of internode elongation imposed by activation of the GVG transcription factor could be

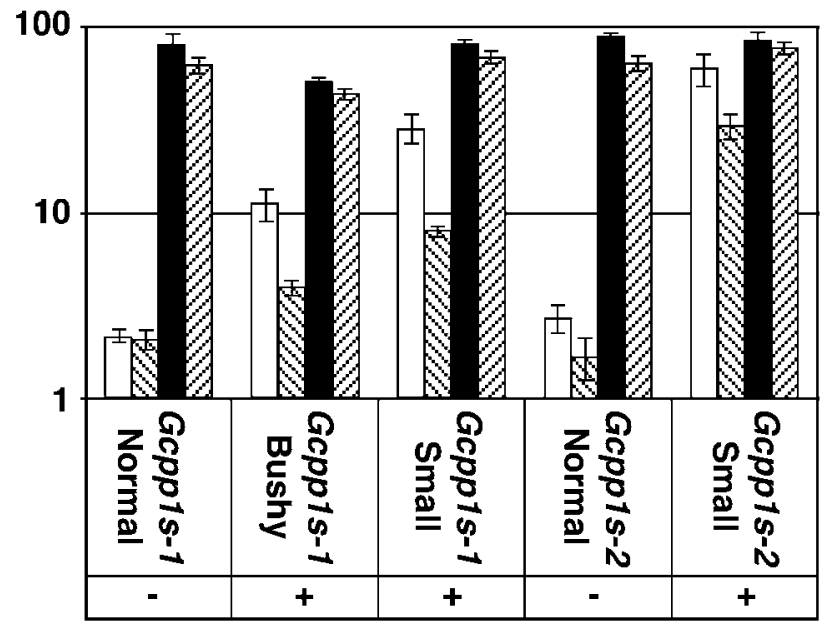

Fig. 3. Ubiquitin normalized total Ljcppl and GVG expression levels in two independent Gcppls plant lines. Plants were harvested at day 30. White bars and bars hatched from left downward: Ljcppl expression. Black bars and bars hatched from left upward: GVG expression. Solid bars: expression in roots. Hatched bars: expression in shoots. +: Plants induced with $30 \mu \mathrm{M}$ DEX at day 4. -: Mock-induced plants. Error bars indicate $95 \%$ confidence interval ( $t$ test). alleviated by application of exogenous gibberellin. Plants were grown for 70 days in the presence of DEX. At this age, they displayed very clear small or bushy phenotypes. Treatment of bushy plants with gibberellic acid $\left(\mathrm{GA}_{3}\right)$ at day 70 resulted in a dramatic increase of internode length (Fig. 6C and D), whereas there was no change in the appearance of small plants upon $\mathrm{GA}_{3}$ treatment (Fig. 6E and F). Treatment of wt plants with $\mathrm{GA}_{3}$ resulted in a small increase in internode length (Fig. 6A and B).

In roots, activation of the GVG system causes uncontrolled proliferation of pericycle cells, death of cortical cells, and starch accumulation.

During investigation of Gcppla plants, an abnormal root surface was noticed. In the affected areas, cell files appeared disrupted and the root was swollen (Fig. 7B). In extreme cases, the root was more than twice as thick as usual and the root surface was completely irregular (Fig. 7C and D). The Gcppla-1 line displayed a severe stunted shoot phenotype and a mildly disordered root phenotype, whereas the opposite was true for the Gcppla-2 line. The lack of correspondence between root and shoot phenotype seen, and the fact that the disordered root phenotype had been observed only in transgenic lines containing GVG-Ljcppl constructs, led us to believe that the disordered phenotype was cppl dependent, and we studied the phenotype in more detail by microscopy.

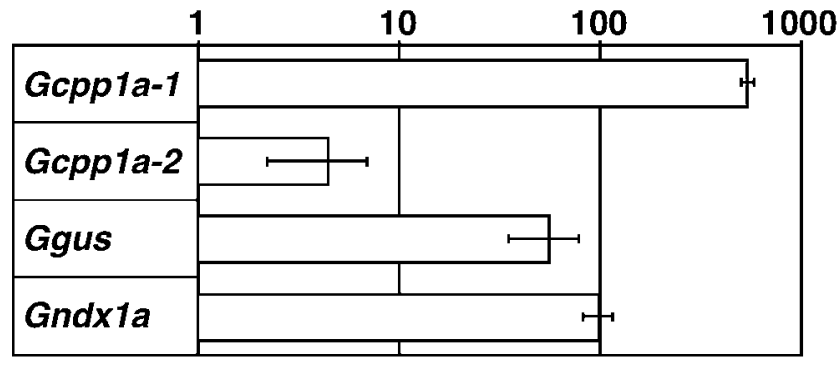

Fig. 4. GVG RNA levels normalized against ATP-synthase RNA levels For each plant line, RNA was extracted from roots of a pool of 21-dayold plants. Error bars indicate $95 \%$ confidence interval ( $t$ test).

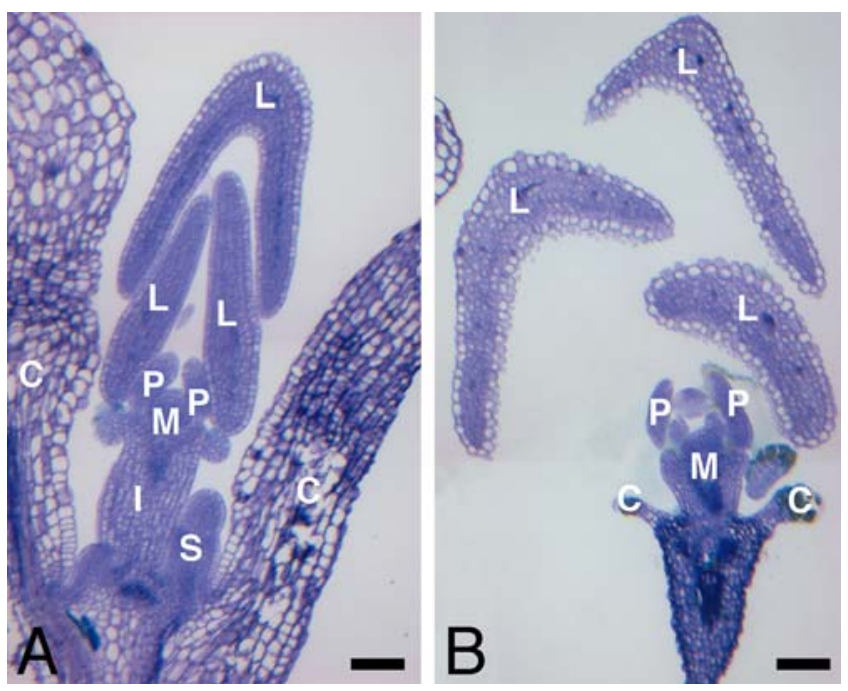

Fig. 5. Toluidine blue-stained sections of Lotus japonicus shoot apical meristems viewed by bright field microscopy. A, Wild-type plant (5 days old). B, Gcpp1s plant (33 days old) induced with dexamethasone at day 4. The overall size of the plants shown in A and B is the same. C: Cotyledons, M: shoot apical meristem, L: developing true leaf, I: internode, S: side shoot, P: leaf primordium. In B, five-leaf primordia are seen in the area marked with two Ps. Bar is $100 \mu \mathrm{m}$. 
The disturbance visible on the surface of the root was caused by excessively proliferating pericycle cells (Fig. 8B and C), cyan blue staining dying cortical cells (Fig. 8B), and the empty spaces left by already deceased cortical cells (Fig. 8F). Furthermore, the affected roots all showed increased deposition of starch in both cortical and pericycle cells but not in endodermal cells (Fig. 8B and C).

In a second round of screening plant lines containing Ggus or Gndxla constructs, roots were found with similar surface defects as those seen in the Gcppla plants. These roots also showed cyan blue staining, dying cortical cells, more than one file of pericycle cells, and increased starch deposition (Fig. 8D and E). Therefore, the effect cannot be ascribed to LjCPP1 function.

The characteristics described were seen in both rhizobiuminoculated and uninoculated roots. Also, functional nodules were observed even on roots displaying severe defects.

\section{DISCUSSION}

Neither the shoot nor the root phenotype observed was linked to expression of a specific transgene. Therefore, the phenotypes described in this study were most likely caused by the GVG inducible system itself. However, the most severe phenotypes in both root and shoot were observed in Gcppla plants, and we cannot rule out the possibility that expression of Ljcppl antisense RNA compounds the GVG phenotype.

\section{The level of GVG-controlled transgene expression is a more reliable indicator of GVG-related phenotypes than the GVG expression level.}

A high level of GVG expression seems a prerequisite for a severe shoot phenotype in L. japonicus because, so far, complete shoot growth arrest has been observed only in plants with a high level of GVG expression. However, this study has shown that plants with similar GVG expression levels can differ greatly in the severity of GVG-related phenotypes. Lack of coherence between GVG expression level and plant phenotype also was reported in an Arabidopsis spp. study where a modified version of the GVG system was used (Sanchez and Chua 2001). On the other hand, transgene expression from the GVGcontrolled promoter did show a correlation with shoot phenotype in both Ggus and Gcppls lines. Such a correlation previously has been reported in an Arabidopsis spp. study (Kang et al. 1999). The expressed transgene is not responsible for the phenotype seen; therefore, it is most likely that the shoot phenotype reflects the amount of active GVG in the nucleus. Thus, a high amount of active GVG in the nucleus would lead to both transcription of the desired transgene and the unwanted disturbance of plant growth. The severity of the GVG phenotype observed has been reported to depend on the concentration of DEX used for induction (Kang et al. 1999; Ouwerkerk et al. 2001). Furthermore, increasing DEX concentration beyond what was necessary to achieve maximum transcription of the transgene further aggravated the phenotype (Ouwerkerk et al. 2001), suggesting that a higher concentration of inducer was able to increase the amount of aberrant GVG activity in the nucleus.

\section{There is not always a direct correlation between shoot and root phenotype.}

Whereas the shoot phenotype may well be a reliable measure of GVG activity in the nucleus, the situation is more complex with regards to the root phenotype observed in L. japonicus.

The Gcpp1a-2 line, which had the lowest GVG-expression level and the least severe shoot phenotype, still showed an obvious disordered root phenotype. Originally, this led us to believe that the root phenotype was independent of the shoot phenotype and of the GVG system. We were forced to reconsider, though, when we found the phenotype in plants that were not expressing antisense Ljcpp1 and could neither detect GVG-
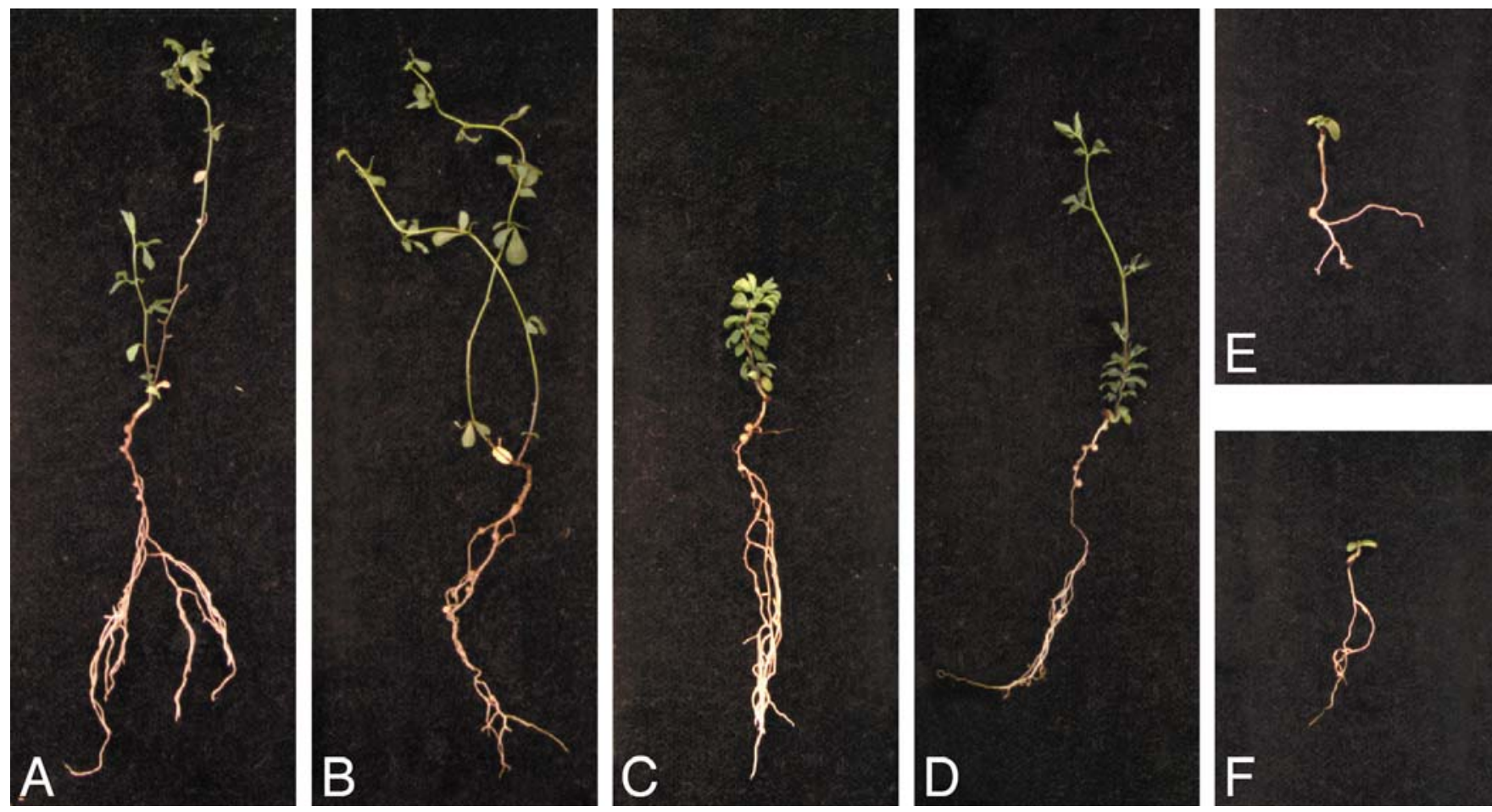

Fig. 6. Gibberellic acid $\left(\mathrm{GA}_{3}\right)$ effect on GVG-induced shoot phenotype. Lotus japonicus plants (90 days old) inoculated with rhizobium at day 4 . A, Wildtype plant treated with dexamethasone (DEX) at day 4 . B, Wild-type plant treated with DEX at day 4 and with $\mathrm{GA}_{3}$ at day 70 . C through $\mathbf{F}, G c p p 1 s$ plants. C, Bushy plant treated with DEX at day 4. D, Bushy plant treated with DEX at day 4 and with $\mathrm{GA}_{3}$ at day 70 . E, Small plant treated with DEX at day 4 . F, Small plant treated with DEX at day 4 and with $\mathrm{GA}_{3}$ at day 70 . 
induced transgene expression nor reduction in endogenous Ljcpp1 mRNA levels in Gcppla-2 plants (data not shown). Instead, we conclude that there must be different thresholds for GVG-induced phenotypes in shoot and root. Sequestration of the hormone-binding domain of Xenopus laevis GR by plastids in the shoot of Arabidopsis spp. has been described recently (Brockmann et al. 2001). The targeting of GR to plastids greatly reduced the amount of GR translocated to the nucleus. This effect was not seen in roots and, consequently, more GR was found in root nuclei than shoot nuclei. If a similar interaction is taking place between plastids in L. japonicus shoots and the rat GR of the GVG transcription factor, this offers a possible explanation of the different properties of the root and shoot phenotypes. Though a severe root phenotype was observed in combination with a mild shoot phenotype in the Gcppla-2 line, the severity of root and shoot phenotype were coupled within individual lines and no lines were found that displayed a root phenotype without showing stunting of the shoot to some degree. Thus, it is possible that the Gcppla-2 line represents a special case, and that lack of correlation between shoot and root phenotype is not a general feature of the GVG expression system in L. japonicus.

\section{The root phenotype is associated with lateral root initiation.}

The disordered root phenotype was not seen in all plants exhibiting a stunted shoot phenotype and, in many cases, it was limited to certain regions of the root. This indicates that even if induction of the GVG system is necessary for the root phenotype, it is not sufficient.

Disturbances in the roots often were seen at sites of lateral root emergence, and misshapen lateral root primordia, which caused swelling of the main root at the site of emergence, also were observed. Considering that the most notable feature of the root phenotype was the uncontrolled proliferation of pericycle cells, these data suggest that lateral root initiation could be the factor needed in conjunction with the GVG system to produce a visible root phenotype. The death of cortical cells seen could be a result of pericycle cell activation, because programmed cell death in roots of soybean has been observed in connection with the emergence of lateral roots (Kosslak et al. 1997).

\section{Deficiency in auxin transport or sensitivity can explain both root and shoot phenotypes.}

The plant hormone auxin is involved in a wide variety of plant developmental processes. The topic has been reviewed recently (Friml 2003; Ross et al. 2001; Vogler and Kuhlemeier 2003). In the shoot, apical dominance is regulated by auxin (Booker et al. 2003) and auxin produced in the apical meristem is necessary to maintain $\mathrm{GA}_{1}$ biosynthesis and internode elongation (Ross et al. 2000). In roots, application of auxin promotes lateral root formation (Laskowski et al. 1995), whereas treatment with $N$-1-naphthylphtalamic acid (NPA), an auxin transport inhibitor, arrests lateral root development (Casimiro et al. 2001). Mutants, such as slr-1 and auxl, that are affected in their auxin response (Fukaki et al. 2002; Marchant et al. 2002) also show reduced lateral root formation. Furthermore, in cultured bright yellow-2 tobacco cells, auxin has been shown to influence amyloplast development and starch accumulation by regulating the expression of genes required for starch biosynthesis (Miyazawa et al. 1999).

By application of the bioactive gibberellin $\mathrm{GA}_{3}$ (Hedden and Phillips 2000), we have shown that plants with a bushy phenotype are gibberellin deficient but not gibberellin insensitive. In bushy plants, we also have observed activation of resting buds, indicating a reduced apical dominance (data not shown). We suggest that the gibberellin deficiency and reduced apical dominance in bushy plants could be the result of a decreased sensitivity to auxin or lack of auxin transport from the apical meristem. A disturbed sensitivity to, or transport of, auxin also might lead to a perturbation in the pericycle cell division pattern during lateral root formation and to increased starch deposition, causing the root phenotype observed in L. japonicus. Offering credibility to this hypothesis is the fact that roots of small plants, in contrast with wt plants, did not respond with swelling of the root tip when treated with the synthetic auxin 2,4-dichlorophenoxyacetic acid (data not shown).

Whilst not directly linked to auxin action, an Arabidopsis marker line LRB10 (lateral root base) (Malamy and Benfey 1997 ) is interesting with respect to the L. japonicus root phenotype. This marker line does not express gus in the primary root tip at all; whereas gus is expressed in all cells of young lateral root primordia and, later, only the cells in the periphery of the primordium. A gene with such an expression pattern could delimit the area of activated pericycle cells during lateral root formation, and disruption of the expression pattern could lead to excess proliferation of pericycle cells, as seen in the present root phenotype.

\section{Is the GVG inducible system useful in the model legume $L$. japonicus?}

Adverse effects of the GVG system have been described in Arabidopsis spp. (Kang et al. 1999; Yamamoto et al. 2001; Zuo and Chua 2000), rice (Ouwerkerk et al. 2001), and L. japonicus (this study). In all cases, the phenotype observed was one of growth retardation. In rice, mild and severe phenotypes (Ouwerkerk et al. 2001), reminiscent of the small and bushy phenotypes in L. japonicus, were seen. In tobacco, no detrimental effect of the GVG system has been reported, to our knowledge. Despite growth defects in several plant systems, the GVG system offers two desirable properties for an inducible system - high inducibility and tight control — and it has been used successfully in Arabidopsis spp. to uncover functions of different genes (Gray et al. 1999; Pautot et al. 2001; Yamamoto et al. 2001; Yoshizumi et al. 1999) as well as for regeneration of transformed plants (Kunkel et al. 1999).
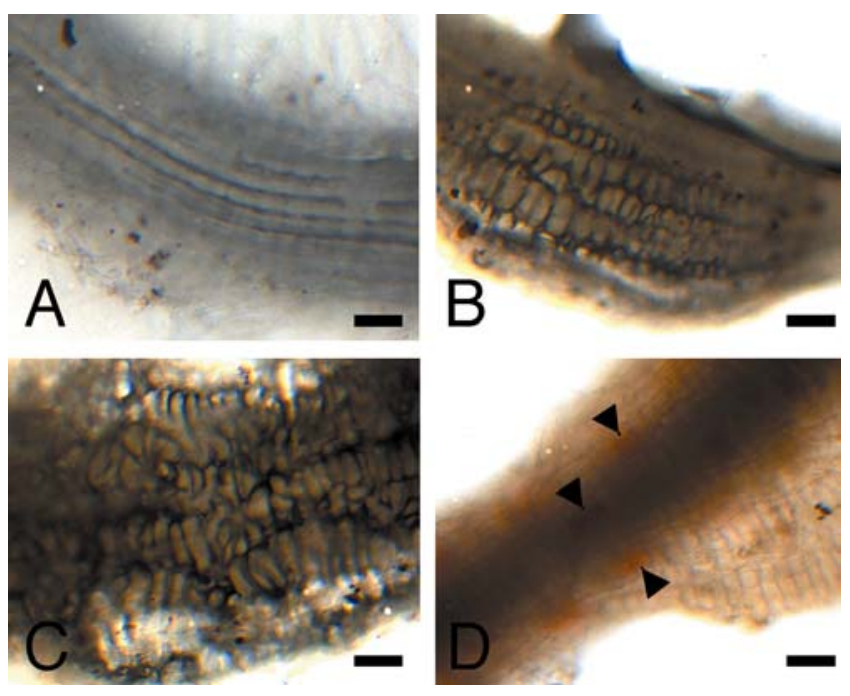

Fig. 7. Lotus japonicus roots viewed by bright field microscopy. A, Wildtype plant (26 days old) supplied with $10 \mathrm{mM} \mathrm{KNO}_{3}$ at day 4. Cell files are regular. B, Gcppla-1 plant (34 days old) induced with dexamethasone (DEX) at day 14 and inoculated with rhizobium at day 21. Root is bloated and cell files are irregular. C and D, Gcppla-2 (T2 generation) plant (53 days old) induced with DEX at day 4 and inoculated with rhizobium at day 21. Cells are improperly aligned and cell shape is altered. D, Root cleared with $70 \%$ ethanol. Brown, dying cortical cells (arrowheads) are visible. Root surface is irregular. Bar is $100 \mu \mathrm{m}$. 

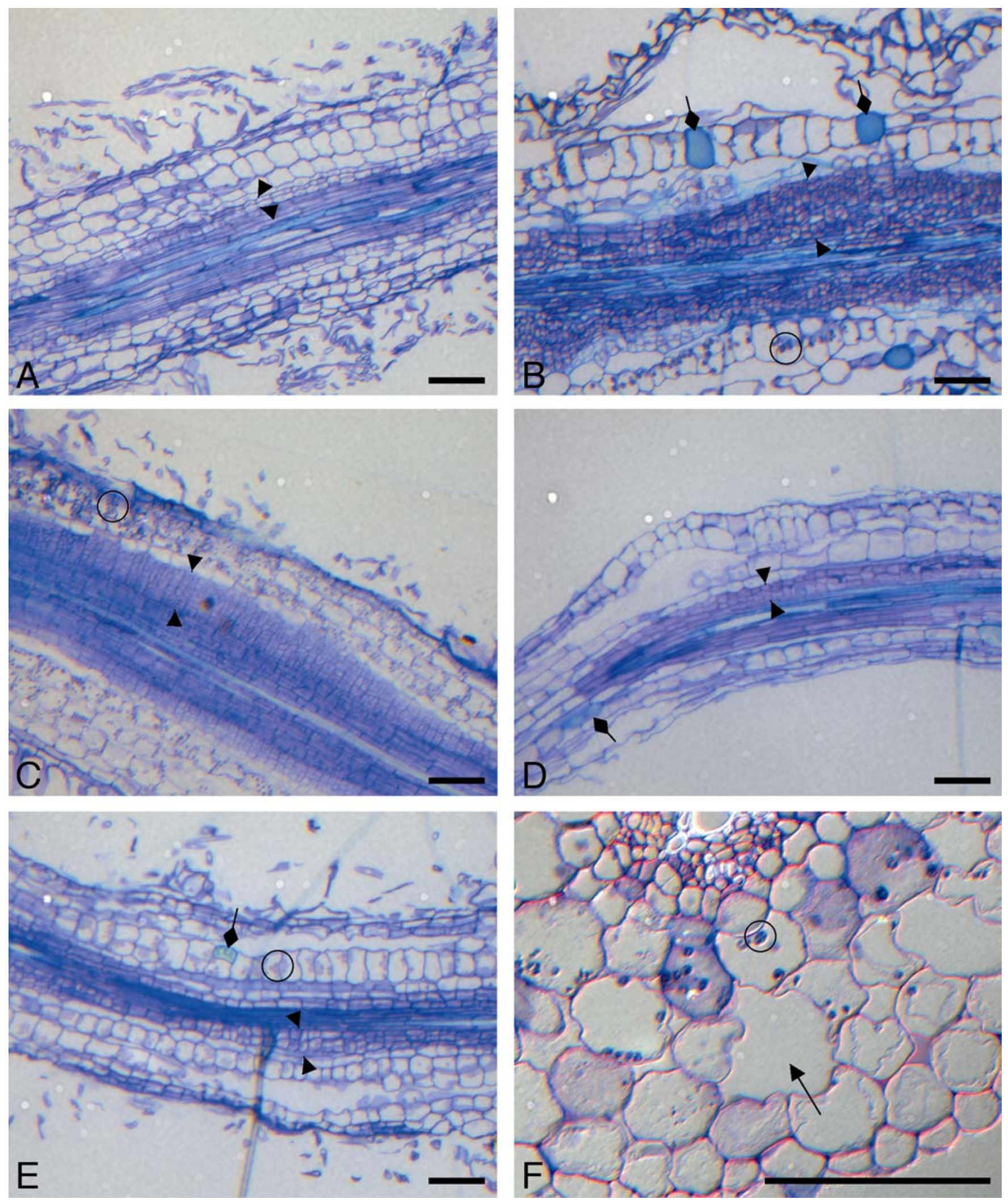

Fig. 8. Sections of Lotus japonicus roots viewed by bright field microscopy. A, Wild-type root (26 days old). B, Gcppla-2 (T2 generation) root (53 days old). C, Gcppla-2 root (26 days old). D, Ggus root (24 days old). E, Gndxla root (27 days old). A through E, Longitudinal sections. F, Gcppla-1 root (26 days old). Cross section viewed by differential interference contrast microscopy. Arrow points to an empty space between the cells. Pericycle cells are found between the arrowheads. Diamond arrows point to cyan blue staining, dying cortical cells. Circles enclose starch grains. Bar is $100 \mu \mathrm{m}$. Sections are stained with toluidine blue and iodine. 
Speculations as to which part of the chimeric GVG transcription factor is causing the defects have been put forward. It was suggested that binding of the transcription factor to ciselements in the plant genome or unspecific transactivating activity of the VP16 domain might be the culprit (Kang et al. 1999). The VP16 domain also has been used in an analogous inducible system, the XVE system (Zuo et al. 2000), which now offers an alternative to the GVG system. Here, no defects were seen, and it was concluded that VP16 alone could not be responsible for the defects seen with the GVG system. Expression of a GAL4-VP16 protein in Arabidopsis spp. did not affect growth (Ouwerkerk et al. 2001) and it seems that inclusion of the rat GR is necessary for producing growth defects. The molecular background for the defects caused by the GVG protein remains unclear; however, our results suggest that auxin signaling somehow is affected.

Successful use of the GVG system requires either that the GVG background phenotype can be avoided or that the phenotype of the gene of interest can be distinguished clearly from the GVG background phenotype. Avoiding the adverse effects of the GVG system by selecting plant lines with low or moderate levels of GVG expression has been suggested (Kang et al. 1999; Zuo and Chua 2000). There is no indication, however, that this is possible in L. japonicus. The T3 generation Gcppla-2 plants had the lowest GVG expression measured so far. Still, they displayed GVG-related phenotypes in both root and shoot, whereas no expression of the transgene could be detected (data not shown). Thus, the threshold for induction of GVG-related phenotypes might be lower than that for useful transgene expression.

If the adverse phenotype cannot be completely avoided, appropriate control lines must be selected. The suggestion to use empty vector control lines with a level of GVG expression similar to that of the lines of interest (Kang et al. 1999; Zuo and Chua 2000) does not ensure the same degree of GVG phenotype in lines of interest and controls, because lines with similar GVG expression levels can have very different phenotypes. What is needed are empty vector controls with the same amount of active GVG in the nucleus as the lines of interest. If the same sufficiently long 5' untranslated region (UTR) were transcribed from the GVG-controlled promoter in both lines of interest and controls, the amount of this $5^{\prime}$ UTR could be monitored by QPCR. It should provide a reliable measurement of GVG activity in the nucleus, as long as inducer concentration is kept within the linear response range for induction of transgene expression. Appropriate controls then could be selected.

Another way to ensure suitable control lines would be to divide the GVG system onto two vectors. The first vector containing the GVG transcription factor would be transformed into plants. These plants then could be used as controls or transformed with a second vector carrying the gene of interest under control of the GVG-responsive promoter. This approach would ensure identical GVG activity levels in controls and lines of interest, provided that the transformation procedure does not alter GVG activity in the double-transformed plants.

A detailed knowledge of the nature of the GVG phenotype would make it easier to distinguish between artifactual and real phenotypes, and such a description is now available for $L$. japonicus. However, there are several drawbacks. The suggested effect on auxin signaling makes it difficult to predict the effect of the GVG system in combination with expression of a transgene. Also, the accumulation of starch grains in roots indicates that plant metabolism is altered. A further concern is the effect on nodulation. When plants were inoculated with rhizobium and induced with DEX at the same time, even plants with severe root and shoot phenotypes were able to form nodules. On the other hand, a reduced nodule formation was observed when Gccpls plants were induced with DEX 7 days prior to inoculation with rhizobium (data not shown). So far, we have been unable to ascertain whether this effect is due to expression of Ljcppl or caused by the GVG system. Until this issue has been resolved, and a satisfactory way of selecting empty vector control lines has been found, we cannot recommend the GVG system for use in the model legume L. japonicus.

\section{MATERIALS AND METHODS}

Constructs for inducible expression.

The full-length Ljcpp1 cDNA was cloned into the pCR IITOPO vector (Invitrogen, Carlsbad, CA, U.S.A.). To make the Gcppls construct, Ljcppl cDNA was excised with SpeI and XhoI, blunt ended, and inserted into the XhoI blunt-ended pTA7002 vector. The Gcppla construct was made by excising Ljcppl cDNA with SpeI and XbaI and ligating it into a SpeI cut pTA7002 vector.

Construction of the GVG-Ljndx constructs has been described previously (Gronlund et al. 2003).

gus was excised with XhoI and BamHI from pSLJ4K1 (Jones et al. 1992), cloned into the pBluescript KS+ vector (Stratagene, La Jolla, CA, U.S.A.), and transferred to pTA7002 with XhoI and SpeI to make the Ggus construct.

\section{RNA extraction and QPCR experiments.}

RNA was extracted from L. japonicus roots with the Nucleospin RNA plant kit (Macherey-Nagel, Düren, Germany) following the manufacturer's instructions.

The following primers were used in QPCR experiments: Ljcppl endogenous RNA, forward 5'CAGTCACTGTAGGGT TTAATTCTTCTTC3', reverse 5'CCTCATCGGAGATAAATT GCTG3'; total amount of Ljcppl mRNA, forward 5'GAATGA TGATCCTGGTGATAAAGC3', reverse 5'CCATGGTACTTC AGACTAGCCTC3'; transgene expression from the Gcppla construct, forward 5'CGCTGAAGCTAGTCGACTCTAG3', reverse 5'TGACTTATTGAAGTTATGACTGCATC3'; GVG expression level, forward 5'GAAGCTACTGTCTTCTATCGA ACAAGC3', reverse 5'CAACATGTCCAGATCGAAATCGT CTAG3'; ubiquitin, forward 5'TCAAGGCTAAGATCCAAGA CAAG3', reverse 5'TCACGTTGTCAATGGTGTCAG3'; and ATP-synthase, forward 5'CAATGTCGCCAAGGCCCATGGT G3', reverse 5'AACACCACTCTCGATCATTTCTCTG3'.

Reactions with primers for Ljcppl endogenous RNA, total amount of Ljcppl mRNA, and ubiquitin were performed with the Lightcycler RNA Amplification Kit, SYBR Green I (Roche, Rotkreuz, Switzerland). In the other reactions, the QuantiTect SYBR Green RT-PCR Kit (Qiagen, Hilden, Germany) was used. All measurements were made using the Lightcycler (Roche) thermal cycler. A dilution standard curve was included in each run for each set of primers. Two to five biological and two to five mechanical replicates were performed for each sample. Relative values for each sample were obtained by use of the dilution standard curves.

\section{Plant growth and transformation.}

Plants were transformed and grown as previously described (Gronlund et al. 2003). Plants were watered with a $30 \mu \mathrm{M}$ DEX solution or sprayed to run-off with a $50 \mu \mathrm{M} \mathrm{GA}_{3}$ solution at the time indicated.

\section{Detection of gus expression.}

GUS activity was assayed in whole plants in a buffer containing $50 \mathrm{mM}$ sodium phosphate $(\mathrm{pH} 7.0), 10 \mathrm{mM}$ EDTA, $20 \%$ (wt/vol) methanol, and $0.05 \%$ (wt/vol) 5-bromo-4-chloro3 -indolyl $\beta$-X-glucoronide (BioSynth AG, Staad, Switzerland) for 16 hours at $37^{\circ} \mathrm{C}$. 


\section{Microscopy.}

$L$. japonicus roots were cut into $\approx 5$-mm pieces, vacuum infiltrated for $30 \mathrm{~min}$ in fixing solution $(1.5 \%$ paraformaldehyde, $2.5 \%$ glutaraldehyde, $0.1 \mathrm{M}$ sodium phosphate buffer, $\mathrm{pH} 7.2$ ), and left in fixing solution overnight at $4^{\circ} \mathrm{C}$. Samples then were embedded in Technovit 7100 (Heraeus Kulzer, Wehrheim, Germany) according to the manufacturer's instructions. Embedded tissue was sectioned with a Leica RM 2045 rotary microtome (Leica, Wetzlar, Germany). Sections were stained with $0.1 \%$ toluidine blue for $2 \mathrm{~min}$, then rinsed with water and ethanol. Root sections were further stained for $5 \mathrm{~min}$ in a 1:50 dilution of an iodine potassium iodide solution (Riedel-de Haen, Seelze, Germany) and rinsed with water. Sections were mounted with Eukitt (Electron Microscopy Sciences, Fort Washington, PA, U.S.A.) and were viewed with an Axioplan2 (Carl Zeiss Microscopy, Jena, Germany) microscope fitted with an AxioCam (Carl Zeiss Micro-Imaging, Thornwood, NY, U.S.A.) camera.

\section{ACKNOWLEDGMENTS}

Research on this project was funded by EU TMX ERBF MRXCT 980243. We thank N.-H. Chua, The Rockefeller University, New York, for providing the pTA7002 vector, and L. Krussel for the primers for ubiquitin amplification.

\section{LITERATURE CITED}

Aoyama, T., and Chua, N. H. 1997. A glucocorticoid-mediated transcriptional induction system in transgenic plants. Plant J. 11:605-612.

Booker, J., Chatfield, S., and Leyser, O. 2003. Auxin acts in xylem-associated or medullary cells to mediate apical dominance. Plant Cell 15:495-507.

Brockmann, B., Smith, M. W., Zaraisky, A. G., Harrison, K., Okada, K., and Kamiya, Y. 2001. Subcellular localization and targeting of glucocorticoid receptor protein fusions expressed in transgenic Arabidopsis thaliana. Plant Cell Physiol. 42:942-951.

Casimiro, I., Marchant, A., Bhalerao, R. P., Beeckman, T., Dhooge, S., Swarup, R., Graham, N., Inze, D., Sandberg, G., Casero, P. J., and Bennett, M. 2001. Auxin transport promotes Arabidopsis lateral root initiation. Plant Cell 13:843-852.

Cvitanich, C., Pallisgaard, N., Nielsen, K. A., Hansen, A. C., Larsen, K., Pihakaski-Maunsbach, K., Marcker, K. A., and Jensen, E. O. 2000. CPP1, a DNA-binding protein involved in the expression of a soybean leghemoglobin c3 gene. Proc. Natl. Acad. Sci. U.S.A. 97:8163-8168.

Friml, J. 2003. Auxin transport-shaping the plant. Curr. Opin. Plant Biol. 6:7-12.

Fukaki, H., Tameda, S., Masuda, H., and Tasaka, M. 2002. Lateral root formation is blocked by a gain-of-function mutation in the SOLITARYROOT/IAA14 gene of Arabidopsis. Plant J. 29:153-168.

Gray, W. M., del Pozo, J. C., Walker, L., Hobbie, L., Risseeuw, E., Banks, T., Crosby, W. L., Yang, M., Ma, H., and Estelle, M. 1999. Identification of an SCF ubiquitin-ligase complex required for auxin response in Arabidopsis thaliana. Genes Dev. 13:1678-1691.

Gronlund, M., Gustafsen, C., Roussis, A., Jensen, D., Nielsen, L. P., Marcker, K. A., and Jensen, E. O. 2003. The Lotus japonicus ndx gene family is involved in nodule function and maintenance. Plant Mol. Biol. 52:303-316.
Handberg, K., and Stougaard, J. 1992. Lotus japonicus, an autogamous, diploid legume species for classical and molecular genetics. Plant J. 2:487-496.

Hedden, P., and Phillips, A. L. 2000. Gibberellin metabolism: new insights revealed by the genes. Trends Plant Sci. 5:523-530.

Jones, J. D., Shlumukov, L., Carland, F., English, J., Scofield, S. R., Bishop, G. J., and Harrison, K. 1992. Effective vectors for transformation, expression of heterologous genes, and assaying transposon excision in transgenic plants. Transgenic Res. 1:285-297.

Jorgensen, J. E., Gronlund, M., Pallisgaard, N., Larsen, K., Marcker, K. A., and Jensen, E. O. 1999. A new class of plant homeobox genes is expressed in specific regions of determinate symbiotic root nodules. Plant Mol. Biol. 40:65-77.

Kang, H. G., Fang, Y., and Singh, K. B. 1999. A glucocorticoid-inducible transcription system causes severe growth defects in Arabidopsis and induces defense-related genes. Plant J. 20:127-133.

Kosslak, R. M., Chamberlin, M. A., Palmer, R. G., and Bowen, B. A. 1997. Programmed cell death in the root cortex of soybean root necrosis mutants. Plant J. 11:729-745.

Kunkel, T., Niu, Q. W., Chan, Y. S., and Chua, N. H. 1999. Inducible isopentenyl transferase as a high-efficiency marker for plant transformation. Nat. Biotechnol. 17:916-919.

Laskowski, M. J., Williams, M. E., Nusbaum, H. C., and Sussex, I. M. 1995. Formation of lateral root meristems is a two-stage process. Development 121:3303-3310.

Malamy, J. E., and Benfey, P. N. 1997. Organization and cell differentiation in lateral roots of Arabidopsis thaliana. Development 124:33-44.

Marchant, A., Bhalerao, R., Casimiro, I., Eklof, J., Casero, P. J., Bennett, M., and Sandberg, G. 2002. AUX1 promotes lateral root formation by facilitating indole-3-acetic acid distribution between sink and source tissues in the Arabidopsis seedling. Plant Cell 14:589-597.

Miyazawa, Y., Sakai, A., Miyagishima, S., Takano, H., Kawano, S., and Kuroiwa, T. 1999. Auxin and cytokinin have opposite effects on amyloplast development and the expression of starch synthesis genes in cultured bright yellow-2 tobacco cells. Plant Physiol. 121:461-469.

Ouwerkerk, P. B., de Kam, R. J., Hoge, J. H., and Meijer, A. H. 2001. Glucocorticoid-inducible gene expression in rice. Planta 213:370-378.

Pautot, V., Dockx, J., Hamant, O., Kronenberger, J., Grandjean, O., Jublot, D., and Traas, J. 2001. KNAT2: evidence for a link between knotted-like genes and carpel development. Plant Cell 13:17191734.

Ross, J. J., O’Neill, D. P., Smith, J. J., Kerckhoffs, L. H., and Elliott, R. C. 2000. Evidence that auxin promotes gibberellin A1 biosynthesis in pea. Plant J. 21:547-552.

Ross, J. J., O’Neill, D. P., Wolbang, C. M., Symons, G. M., and Reid, J. B. 2001. Auxin-gibberellin interactions and their role in plant growth J. Plant Growth Regul. 20:336-353.

Sanchez, J. P., and Chua, N. H. 2001. Arabidopsis PLC1 is required for secondary responses to abscisic acid signals. Plant Cell 13:1143-1154.

Vogler, H., and Kuhlemeier, C. 2003. Simple hormones but complex signalling. Curr. Opin. Plant Biol. 6:51-56.

Yamamoto, Y. Y., Deng, X. W., and Matsui, M. 2001. Cip4, a new cop1 target, is a nucleus-localized positive regulator of Arabidopsis photomorphogenesis. Plant Cell 13:399-411.

Yoshizumi, T., Nagata, N., Shimada, H., and Matsui, M. 1999. An Arabidopsis cell cycle-dependent kinase-related gene, CDC2b, plays a role in regulating seedling growth in darkness. Plant Cell 11:1883-1896.

Zuo, J., and Chua, N. H. 2000. Chemical-inducible systems for regulated expression of plant genes. Curr. Opin. Biotechnol. 11:146-151.

Zuo, J., Niu, Q. W., and Chua, N. H. 2000. Technical advance: An estrogen receptor-based transactivator XVE mediates highly inducible gene expression in transgenic plants. Plant J. 24:265-273. 